\title{
B19 - DIGESTÃo ENZIMÁTICA DE ANTICORPOS MONOCLONAIS MURINOS ANTI-PBP2a DE Staphylococcus aureus RESISTENTES À METICILINA (MRSA) PARA OBTENÇÃO DE FRAGMENTOS F(ab')2
}

$\underline{\text { Anna Erika Vieira de Araujo }}{ }^{1}$, Natália Plínio de Souza ${ }^{1}$, Luis Vidal Conde ${ }^{1}$, Lucas Almeida Machado ${ }^{1}$, Álvaro Paiva Braga de Sousa ${ }^{1}$, José Procópio Moreno Senna ${ }^{1}$.

${ }^{1}$ Bio-Manguinhos, Fundação Oswaldo Cruz, Vice-diretoria de Desenvolvimento Tecnológico, Programa de Biofármacos Laboratório de Tecnologia Recombinante, Rio de Janeiro, Brasil.

INTRODUÇÃO: Infecções causadas por Staphylococcus aureus resistentes à meticilina (MRSA) são um problema de saúde mundial, especialmente devido à dificuldade de tratamento, alto grau de virulência e elevada morbidade associada. Por ser uma bactéria multirresistente, estratégias alternativas de tratamento têm sido pesquisadas, como, por exemplo, imunoterapias passiva e ativa, porém até agora nenhuma obteve sucesso. Diante desse quadro, Bio-Manguinhos vem desenvolvendo um anticorpo monoclonal para o tratamento de infecções causadas por MRSA tendo como alvo a PBP2a, uma proteína de baixíssima afinidade por $\beta$-lactâmicos encontrada exclusivamente em cepas de MRSA. Fragmentos de anticorpos, contendo apenas as duas porções Fab', são descritos na literatura como ferramentas imunoquímicas, reagentes de diagnóstico e terapêutica, apresentando uma farmacocinética mais rápida, menor imunogenicidade e maior poder de penetração em tecidos quando comparados à $\mathrm{IgG}$.

OBJETIVOS: Digestão enzimática de anticorpos monoclonais murinos anti-PBP2a por papaína e pepsina para a obtenção de fragmento $F\left(a b^{\prime}\right)_{2}$ e avaliação da afinidade à $\mathrm{PBP} 2 \mathrm{a}$ do fragmento $\mathrm{F}\left(\mathrm{ab}{ }^{\prime}\right)_{2}$ por ensaios imunoenzimáticos.

METODOLOGIA: Para otimizar a obtenção dos fragmentos $\mathrm{F}\left(\mathrm{ab}{ }^{\prime}\right)_{2}$ por digestão enzimática com papaína e pepsina, foram testadas diversas variáveis, como diferentes proporções enzima/anticorpo, variabilidade nos tempos de digestão e uso de inibidor de proteases (PMSF, para a papaína). As frações $\left.\mathrm{F}(\mathrm{ab})_{2}\right)_{2}$ obtidas foram purificadas através de cromatografia de afinidade utilizando resinas de Proteína A, como a MabSelectSure (GE) e concentradas por unidades filtrantes Amicon ${ }^{\circledR}$ MWCO $50 \mathrm{kDa}$. As amostras de 
anticorpo digerido e suas frações foram submetidas à análise por eletroforese em gel de poliacrilamida (SDS-PAGE) na ausência de agente redutor. Para avaliar a afinidade do fragmento $\mathrm{F}\left(\mathrm{ab}{ }^{\prime}\right)_{2}$ pela PBP2a foram realizados ensaios imunoenzimáticos, como ELISA do tipo indireto e Western Blot.

RESULTADOS: A análise dos SDS-PAGE indicaram que foi possível a obtenção e o isolamento de fragmentos $\left.\mathrm{F}(\mathrm{ab})^{\prime}\right)_{2}$ por digestão com papaína e pepsina (banda de aproximadamente $110 \mathrm{kDa}$ ), nas proporções de 1:10 (enzima/anticorpo, p/p) por 30 minutos e com uso de PMSF, para a digestão com a papaína. A digestão com a pepsina apresentou melhor produção de $\left.\mathrm{F}(\mathrm{ab})_{2}\right)_{2}$ com melhor rendimento $(74 \%$, enquanto que a digestão com a papaína obteve 56\%). Os ensaios de ELISA e Blot demonstraram que os $\mathrm{F}\left(\mathrm{ab}^{\prime}\right)_{2}$ não perderam afinidade pela PBP2a, apresentando perfil semelhante ao da IgG, mesmo após o processo de digestão enzimática com pepsina e papaína.

CONCLUSÃO: Foi possível obter o fragmento $\mathrm{F}\left(\mathrm{ab}{ }^{\prime}\right)_{2}$ por digestão enzimática e isolálo por cromatografia de afinidade e ultrafiltração. Tal fragmento apresentou afinidade à PBP2a de forma semelhante à $\mathrm{IgG}$, demonstrando que o processo de digestão enzimática não foi capaz de alterar esse parâmetro. 